\title{
Galactic Structure Toward the Carina Tangent
}

\author{
N. T. Kaltcheva \\ Department of Physics and Astronomy, University of Wisconsin Oshkosh, 800 Algoma \\ Blvd., Oshkosh, WI 54901, USA \\ kaltchev@uwosh.edu \\ and \\ V. K. Golev \\ Department of Astronomy, Faculty of Physics, St Kliment Ohridski University of Sofia, 5 \\ James Bourchier Blvd., BG-1164 Sofia, Bulgaria \\ valgol@phys.uni-sofia.bg
}

\begin{abstract}
This investigation presents a photometric study of the Galactic structure toward the Carina arm tangent. The field is located between $280^{\circ}$ and $286^{\circ}$ galactic longitude and $-4^{\circ}$ to $4^{\circ}$ galactic latitude. All currently available uvby $\beta$ data is used to obtain homogeneous color excesses and distances for more than 260 stars of spectral types $\mathrm{O}$ to $\mathrm{G}$. We present revised distances and average extinction for the open clusters and cluster candidates NGC 3293, NGC 3114, Lodén 46 and Lodén 112. The cluster candidate Lodén 112 appears to be a very compact group at a true distance modulus of $11.06 \pm 0.11$ (s.e.) (1629-80 pc), significantly closer than previous estimates. We found other OB stars at that same distance and, based on their proper motions, suggest a new OB association at coordinates $282^{\circ}<l<285^{\circ},-2^{\circ}<b<2^{\circ}$. Utilizing $\mathrm{BV}$ photometry and spectral classification of the known O-type stars in the very young open cluster Wd 2 we provide a new distance estimate of $14.13 \pm 0.16$ (s.e.) $\left(6698_{-475}^{+512} \mathrm{pc}\right.$ ), in excellent agreement with recent distance determinations to the giant molecular structures in this direction. We also discuss a possible connection between the HII region RCW 45 and the highly-reddened B+ star CPD -55 3036 and provide a revised distance for the luminous blue variable HR Car.
\end{abstract}

Subject headings: Galaxy: structure; open clusters and associations: individual (NGC 3293); open clusters and associations: individual (Lodén 46); open clusters and associations: individual (Lodén 112); open clusters and associations: 
individual (NGC 3114); open clusters and associations: individual (Wd 2); stars: distances; stars: individual (HR Car)

\section{Introduction}

In the fourth Galactic quadrant, the direction toward $282^{\circ}-285^{\circ}$ galactic longitude corresponds to maxima in the thermal radio continuum, Hi and CO emissions (Taylor \& Cordes 1993; Bloemen et al. 1990; Bronfman 1992). Other kinds of tracers also indicate that in this direction the line of sight is tangential to a large segment of the Carina arm. Based on a multiwavelenght study of star-forming complexes, it is found that longitudes $283^{\circ}-284^{\circ}$ correspond to a tangential direction in both the 3-and 4-arm models of the grand design of the MW (Russeil 2003; Vallée 2008).

The OB stars observed in the $283^{\circ}-284^{\circ}$ longitude range delineate a sharp outer edge of the Carina arm at about 2-3 kpc from the Sun (Graham 1970; Kaltcheva \& Scorcio 2010). The field located between the associations in Vela $\left(262^{\circ}<l<268^{\circ}\right)$ and Car OB1 $\left(284^{\circ}<\right.$ $l<288^{\circ}$ ) is not known to be dominated by any prominent OB association (Humphreys 1978; Melnik \& Efremov 1995). The only known SNR in the region is G279.0+1.1 (Duncan et al. 1995).

Local and intermediate-scale features of the Galactic disk, like arm-splitting and branching, are important part of the grand design of the Milky Way (see Russeil (2003) for a recent discussion). This paper is focused on a field between $280^{\circ}$ and $286^{\circ}$ in the Galactic disk with aim to address the present deficiency in the study of the structure toward the edge of the Carina arm. Our study is based on uvby $\beta$ photometric distances and provides new insights on the apparent groupings and layers in this region.

\section{The sample}

The field under consideration is located between $280^{\circ}$ and $286^{\circ}$ galactic longitude and $-4^{\circ}$ and $4^{\circ}$ galactic latitude. All uvby $\beta$ data within this coordinate range was extracted from the catalog of Hauck \& Mermilliod (1998) and combined with the uvby $\beta$ photometry from the catalog of Kaltcheva (2003). The sample contains more than 300 stars of spectral types $\mathrm{O}$ to $\mathrm{G}$ with complete uvby $\beta$ photometry and is listed in Table 1, available only electronically. Fig. 1 presents these stars plotted in Galactic coordinates. All known open clusters and all Lodén cluster candidates are shown as well and are listed in Table 2. 


\section{Calculation of interstellar extinction and distances}

To infer the physical stellar parameters from the photometry, the spectral and luminosity classifications (MK) were extracted from the SIMBAD database and used in conjunction with the classification $\left[c_{1}\right]$ vs. $\left[m_{1}\right]$ diagram (Strömgren 1966) to study the spectral content of the sample. The sample contained about 40 A-F-G type stars of luminosity classes III-II-Ib, which were clearly separated on the $\left[c_{1}\right]$ vs. $\left[m_{1}\right]$ diagram. Since for these types a reliable estimate of distance is not available at present in the uvby $\beta$ system, they were excluded from the following analysis. The location of the rest of the stars on the classification $\left[c_{1}\right]$ vs. $\left[m_{1}\right]$ diagram shows a fair agreement with the SIMBAD MK (Fig. 2, top right panel).

The procedure applied here to obtain the color excesses and stellar distances for the $\mathrm{O}$ and $\mathrm{B}$ stars in the sample is described in detail in Kaltcheva \& Hilditch (2000). The color excesses for LC III, IV and V are obtained via Crawford's (1978) calibration. The calibration by Kilkenny \& Whittet (1985) is used for LC II, Ib, Iab and Ia. We used R=3.2 and $E(B-V)=E(b-y) / 0.74$ to obtain $V_{0}$. The calibration by Balona \& Shobbrook (1984) is utilized for all O-B9 stars to derive the $M_{V}$ values. Since we are dealing with early spectral types, the presence of emission lines in the stellar spectra is the largest source of error in the calculated absolute magnitudes. However, the $\beta$ vs. $c_{0}$ diagram (not shown here) reveals that very few $\mathrm{O}$ and $\mathrm{B}$ stars in the sample deviate from the main sequence and have photometry affected by emission. For all stars with observed $\beta$ values outside the limits of the $M_{V}$ calibration, and all known emission-line stars, $\beta$ calculated from $c_{0}$ was used to obtain $M_{V}$ (cf. for details Balona 1994, Kaltcheva \& Hilditch 2000). Note that this procedure yields distance in excellent agreement with the recalculated Hipparcos data (Kaltcheva \& Makarov 2007). Recently Kaltcheva \& Golev (2011) presented a comparison between the Hipparcos and uvby $\beta$ photometric $M_{V}$ quantified as a function of the spectral sub-type in the B0-B9 range. This comparison was done for field stars with good-quality Hipparcos parallaxes that are subject to relative errors of less than $10 \%$ and shows that the agreement is in place over the entire B-type spectral range.

For the rest of the sample we follow Crawford 1975a, 1979) and separate the A2-F2 stars from the F2-G2 stars according to their $\beta$ indices. All stars with $\beta$ in the range 2.55 to 2.7 we refer to as F-type and all stars with $\beta$ between 2.7 and 2.9 we consider to be A-type. This photometric classification agrees very well with the SIMBAD MK types. To derive the individual color excesses and absolute magnitude for these stellar types, we apply the calibrations of Crawford 1975a, 1979). The calibration of Hilditch et al. (1983) is applied to the A0-A2 stars in the sample, which photometric classification based on the $\left[c_{1}\right]$ vs. $\left[m_{1}\right]$ diagram is also in excellent agreement with the SIMBAD MK types. The photometric data and the derived stellar parameters are summarized in Table 1 which includes the stellar 
identifications, followed by the galactic coordinates, MK type and uvby $\beta$ photometric data, color excess and dereddened photometry, calculated absolute magnitude and true distance moduli. The expected uncertainties in $M_{V}$ are of the order of \pm 0.3 mag for $\mathrm{O}$ and $\mathrm{B}$ types of LC III-V and for A-F V-IV types, and \pm 0.5 mag for B-type super-giants. An uncertainty of $\pm 0.3 \mathrm{mag}$ in $M_{V}$ propagates to an asymmetric error of $-13 \%$ to $+15 \%$, and uncertainties of \pm 0.5 mag result in $-21 \%$ to $+26 \%$ error in the derived distances. Since the photometry used in this paper comes from different sources, the homogeneity of the sample is an important issue. Comparisons of existing uvby $\beta$ data-sets collected by various authors for the field of Carina Spiral Feature present in general a good agreement (see Kaltcheva et al. (2000)). The latter authors estimated that the uncertainty in the calculated stellar distances due to possible systematic deviations in the existing photometric data should not exceed $3-5 \%$.

Note, that in the uvby $\beta$ system both the color excess and absolute magnitude calculations do not rely on a precise determination of spectral type, since the calculations are carried on in the same way for types from O to B9, A0-A2, A2-F2, and F2-G2. Inspecting the reddening free $\left[c_{1}\right]$ vs. $\left[m_{1}\right]$ diagram is sufficient to identify and resolve spectral type misclassifications, but we did not notice any for our sample. However a great care was taken to resolve all cases of suspected luminosity class misclassification since different calibrations are used to calculate the color excesses for different LC types, which is especially important in the O-B9 spectral range. To ensure as proper an LC classification as possible, the database was divided into groups according to the LC available in SIMBAD and each group was considered separately. The reddening free $\left[c_{1}\right]$ vs. $\left[m_{1}\right]$ and $\left[c_{1}\right] / \beta$ diagrams (not shown here) built for each LC were used to examine for possible LC misclassifications and also for stars with $\mathrm{H}_{\beta}$ emission. The individual sources of spectral classification were also considered for all cases of observed inconsistencies, especially the catalogue of Reed (2003) for OB stars and its updates. Again, we did not notice inconsistencies between the LC types listed in SIMBAD and the photometric classification diagrams we built and inspected, or other literature sources of luminosity classification involved in the comparisons.

\section{Photometry-derived results}

The diagrams color excess $E(b-y)$ vs. distance moduli, $V_{0}$ vs. $(b-y)_{0}$ and $M_{V}$ vs. $(b-y)_{0}$ are presented in Fig. 2 and are used to reveal spatially coherent structures in the

studied longitude range. Figures 3, 4 and 5 show the stars with available distances plotted in galactic coordinates. The backgrounds in these figures present the distributions of $H \alpha$, ${ }^{12} \mathrm{CO}(\mathrm{J}=1 \rightarrow 0,115 \mathrm{GHz})$, and dust infrared emission, and are described in the captions of the figures. 
There is a number of papers devoted to star formation in dense ISM in the surroundings of HiI regions, both from theoretical and observational point of view (see, for example, Cichowolski et al. (2009) and the references therein). According to the current theories massive stars tend to stimulate star formation at larger distances, but affect destructively their immediate neighborhood, since they tend to disrupt the parental molecular cloud. It could be seen in Figs. 4 and 5 that the location of the stars in the sample does not correlate with the dense molecular clouds represented by the distribution of the ${ }^{12} \mathrm{CO}$ flux, and with zones of high reddening. On the other hand, the stellar distribution correlates with the location of ionized hydrogen in Fig. 3. Studying the distributions of OB stars together with the distributions of $H \alpha$ (a tracer of ionized hydrogen), ${ }^{12} \mathrm{CO}$ (a tracer for neutral hydrogen and molecular clouds), and dust infrared emission, could give more complete picture of the massive stellar population in the region, the different components of the ISM, and the interactions among them.

\subsection{Lodén 112 and IC 2581}

Lodén 112 is a poor cluster candidate that contains about $10 \mathrm{OB}$ stars. Five of them are included in our sample: HD 300811, HD 300813, HD 300814AB, CPD -56 3492, CPD -56 3496 (see Table 1). These stars form a very compact group at a (median) true DM = 11.06 ( $\pm 0.28 \mathrm{sd} ; \pm 0.12 \mathrm{se})$ and average color excess $E(b-y)=0.5( \pm 0.07 \mathrm{sd} ; \pm 0.03 \mathrm{se})$. For all groups under consideration in this paper we will indicate both the standard deviation (sd) and standard error (se) when providing distance modulus and color excess. When calculating the distance to the groups, the uncertainties will be based on the standard error in the distance modulus. Our distance estimate of $1629_{-80}^{+84} \mathrm{pc}$ for Lodén 112 is significantly smaller than the presently adopted 2500 pc (see for example WEBDA database). The 2500

pc distance is based on the $\mathrm{V}$ vs. B-V diagram of the cluster (Kharchenko et al. 2005). Since all known possible members of Lodén 112 are found in the upper part of the MS, a determination based on the location of these stars on the color-magnitude diagram is difficult. The uvby $\beta$ photometry allows us to obtain the distance to each stars and find an average distance to the cluster. Lodén 112 has been last studied by Lodén (1977) who performed UBV and uvby $\beta$ photometry of stars in the Carina-Crux-Centaurus-Norma region suspected of belonging to poor open clusters or associations. However, the characteristics of these cluster candidates have not been photometrically studied based on uvby $\beta$ photometry. The photometric diagrams and individual stellar distances presented here (Fig. 2) indicate that one of these candidates, Lodén 112, is possibly a physical group. In our sample there are 9 other early B main-sequence stars located at that distance (HD 84361, HD 89174 (both found at the edge of the studied field at galactic longitude approximately $280^{\circ}$ ), HD 88661, HD 
90102, HD 90273, HD 90288AB, HD 90615, CPD -54 3538, CPD -55 3036). Two relatively evolved stars (HD 89714 and HD 90135) are also found in this distance range. For all 16 stars mentioned in this paragraph the (median) true DM is 11.12 ( $\pm 0.6 \mathrm{sd} ; \pm 0.15 \mathrm{se})$.

The open cluster IC 2581 seems to be part of the feature of OB stars mentioned above. The two brightest OB stars of the cluster (HD 90706 and HD 90707) have uvby $\beta$ data available. They are found at an average true $\mathrm{DM}=11.56( \pm 0.63 \mathrm{sd} ; \pm 0.45 \mathrm{se})$, corresponding to a distance 2051 pc (vs. 2446 pc provided by Dias et al. (2003)), and have average color excess $E(b-y)=0.43( \pm 0.02 \mathrm{sd} ; \pm 0.02 \mathrm{se})$. The cluster has been previously studied by Lloyd-Evans (1969) who found DM=12.0 (2500 pc) and Turner (1973) (DM=11.65 (for $\mathrm{R}=3$ ), corresponding to $2140 \mathrm{pc}$ ).

The field considered in this paragraph clearly stands apart from the large Hiı features toward Car OB1 ( $\eta$ Car complex) and contains several smaller but prominent Hir nebulosities, among which RCW 48 and RCW 49 (Fig. 6, top). This field is often called the "preceding end of the Carina complex" (see Lloyd-Evans (1969) for example). It is difficult to judge if the OB stars considered in this subsection are spatially connected to these HiI regions, or are foreground. Note that the distant cluster $\mathrm{Wd} 2(6.7 \mathrm{kpc}$, see 4.6$)$ is found at the center of RCW 49. On the other hand, IC 2581, at only about $2-2.5 \mathrm{kpc}$, seems to be located at the edge of RCW 49, the angular distance between IC 2581 and Wd 2 being about 25 arcmin. IC 2581 is considered of intermediate age and thus should not be involved in the bright nebulosity. However, this may not be true for the two brightest OB stars in the cluster, considered here. Note that it is not certain that these two stars are actually members of the cluster. A loose constraint between 2 and $5 \mathrm{kpc}$ of the distance to RCW 49 has been derived by Tsujimoto et al. (2007) from the mean X-ray luminosity of T-Tauri stars. Ascenso et al. || (2007) proposed $2.8 \mathrm{kpc}$ based on NIR magnitudes and colors of RCW 49 sources on the Henyey track.

For all stars considered in this subsection proper motions are available (Fig. 6, bottom), and are similar for the majority of them. Thus, based on distances and proper motions, we suggest a new $\mathrm{OB}$ association at coordinates $282^{\circ}<l<285^{\circ},-2^{\circ}<b<2^{\circ}$, connected to the compact Lodén 112 group and containing HD 90706 and HD 90707 (probable members of IC 2581). The (median) true DM for all 18 stars studied in this subsection is 11.13 $( \pm 0.59 \mathrm{sd} ; \pm 0.14 \mathrm{se})$, corresponding to $1682_{-104}^{+113} \mathrm{pc}$. Having in mind the independently obtained constraints and estimates of the distance to RCW 49, it is difficult to judge whether this feature of OB stars is connected to the HiI nebulosities seen in this direction or is foreground. 


\subsection{HR Car}

HR Car (HD 90177) is a luminous blue variable known to undergo slow irregular spectrophotometric variations of about $1.5 \mathrm{mag}$ over timescales of months (Carlson \& Henize 1979). The most recent attempt to obtain the distance to HR Car (van Genderen et al. 1991$)$ is based on the reddening-distance method of field stars and yields $5 \pm 1 \mathrm{kpc}$. This estimate matches well the $5.4 \mathrm{kpc}$ distance to the Carina arm obtained kinematically (Hutsemékers \& van Drom 1991). Other existing distance estimates are based on the assumption that HR Car belongs to the Carina complex at the canonical distance of $2.5 \mathrm{kpc}$ (Viotti 1971). However, the line of sight to the Carina complex is tangential to the Carina spiral arm, so the luminous stars seen in this direction may have a very large range in distance (see for example Kaltcheva \& Scorcio (2010)). The Hipparcos distance obtained via the revised parallaxes (van Leeuwen 2007) is $592_{-194}^{+577} \mathrm{pc}$, thus locating the star much closer than any other estimate. We stress however that HR Car lies in a relatively crowded region and this could affect the accuracy of the Hipparcos parallax.

The visual magnitude of HR Car in general varies between 7.6 and 8.6 mag (Parthasarathy et al. 2000). The measured $\beta=2.392$ indicates that the star is observed in emission and a $\beta$ index obtained via $c_{0}$ should be used when calculating the absolute magnitude (see Kaltcheva \& Hilditch (2000)). This yields $M_{V}=-8.3$ mag. The emission usually does not affect the color excess calculation. Here we adopt $E(b-y)=0.912$ and a visual magnitude of 8.076, which provides a distance modulus 12.37, corresponding to $3 \mathrm{kpc}$ (see Table 1). This is the second most reddened star in our sample. We estimate $A_{V}=3.96$ mag (for $R=3.2$ ).

HR Car is variable both in photometry, spectral type and luminosity class. The available MKK classifications indicate spectral type from B2 to B9 and luminosity class I. Since the $(b-y)_{0} \quad$ vs. $c_{0}$ relations used for reddening determination are identical for LC I-II near to the upper part of the MS (Kilkenny \& Whittet 1985), a variable luminosity class should not influence the obtained color excess. As previously mentioned, both color excess and $M_{V}$ determination do not depend of the spectral subtype. In this sense, the variability in spectral classification of HR Car should not influence the obtained distance.

The distance and absolute magnitude of HR Car are important for a variety of reasons, like the theoretical interpretation of this stellar type (van Genderen et al. 1991), studying the spatial distribution of dust around the star (Umana et al. 2009), etc. Apparently an ambiguity in both estimates is still present. However, despite of the peculiarity of this star, our method based of uvby $\beta$ photometry provides reasonable estimates of both quantities and indicates that the currently accepted distance of $5 \mathrm{kpc}$ is overestimated and should be applied with caution. On the other hand, the $M_{V}$ estimate obtain here is in agreement with the one derived by van Genderen et al. (1991). 


\subsection{RCW 45 and CPD -553036}

RCW 45 (BRAN 295) is a rather isolated HiI region (Fig. 6, top), located at coordinates $l=282.13^{\circ}, b=-0.11^{\circ}$, with angular size of 16 arcmin and radial velocity $V_{\mathrm{lsr}}=-9.8 \mathrm{~km}$

$\mathrm{s}^{-1}$. RCW 45 is included in the star-forming field Avedisova 2297, at an accepted distance 6500 pc and angular size 30.25 arcmin.

The distance estimate of $6500 \mathrm{pc}$ is based on the survey of Hi 21-cm emission in the southern Milky Way by McClure-Griffiths et al. (2000). These authors have detected two large shells in the interstellar neutral hydrogen near the Carina tangent, centered at $(l, b)=(277,0)$ and $(l, b)=(280,0)$ that share a common line of sight. The center velocities are $\sim 36 \mathrm{~km} \mathrm{~s}^{-1}$ and $\sim 59 \mathrm{~km} \mathrm{~s}^{-1}$, which puts the shells at kinematic distances of $6.5 \pm 0.9 \mathrm{kpc}$ and at $10 \mathrm{kpc}$, respectively. GSH $277+00+36$ can be classified as a supershell on the basis of its large size and expansion energy. The above authors find evidence for molecular clouds along the supershell's edges, indicating that a star formation may have been initiated by the supershell's expansion. They suggest that the prior interpretation of this large void as an interarm region is inappropriate on the basis of the supershell's chimney and shell-like morphology. The shells should be rather considered interarm voids, as previously suggested by Grabelsky et al. (1987).

The relation of RCW 45 to this supershell is not clear. In their catalog of candidates for Galactic worms (the walls surrounding the superbubbles) Koo et al. (1992) noted that the HiI regions RCW 45 and RCW 46 lie at the base of GW 281.5+1.5, which place them in the eastern edge of the shell (McClure-Griffiths et al. 2000). Note that RCW 45 and RCW 46 do not overlap with the worm but are located at the plane just below the worm candidate (table 5 of Koo et al. (1992)). Also note that RCW 45 is marked with a question mark in their Table 5. All this means that accepting the supershell distance of $6.5 \mathrm{kpc}$ as a distance to RCW 45 has to be done with caution.

CPD - $553036\left(\operatorname{LS~} 1448 ; l=282.166^{\circ}, b=-0.025^{\circ} ; V_{\text {lsr }}\right.$ not available) is located in the direction of RCW 45 and is the most reddened star in our sample. Using UBV photometry, Denoyelle (1977) obtained $E(B-V)=1.62\left(A_{V}=5.18\right.$ for $\left.\mathrm{R}=3.2\right)$ and a distance of 1380 pc. Based on UBV $\beta$ photometry Wramdemark (1980) obtained $A_{V}=5.1$ mag and DM = $9.2(700 \mathrm{pc})$. The star is included in the extensive uvby photometric study of the Luminous Stars in the Southern Milky Way by Kilkenny \& Whittet (1993). Kilkenny (1993) presents $\beta$ photometry of the star and obtains $A_{V}=5.14$ and a distance of $1200 \mathrm{pc}$. In this paper we determine $A_{V}=5.36 \mathrm{mag}$ and $\mathrm{DM}=10.21 \mathrm{mag}$, which corresponds to a distance of 1101 pc.

The high interstellar extinction toward CPD -55 3036 and its proximity to RCW 45 
in terms of Galactic coordinates may indicate a possible relation. However, the uncertainty in the distance of RCW 45 discussed above and the lack of radial velocity measurements for the star would make at this point further conclusions preliminary.

\subsection{Lodén 46}

Lodén (1979) performed uvby $\beta$ of 15 stars of the cluster candidate in field 46 (Lodén 46 ) and estimated a distance of $1.24 \mathrm{kpc}$ and $E(B-V)=0.22$ mag. Lodén 46 is represented in our sample by 9 stars of spectral class A0-A2. We determine a true median DM = 8.67 ( $\pm 1.42 \mathrm{sd} ; \pm 0.47 \mathrm{se}$ ), corresponding to a distance $542 \mathrm{pc}$, in good agreement with the adopted distance of $540 \mathrm{pc}$ (see WEBDA database). The relatively large spread in individual distances for these stars may be due to the fact that the uvby $\beta$ system is not very suitable for the A0-A2 spectral range.

\subsection{NGC 3293 and NGC 3114}

These clusters are studied in details by various authors. Here we present only revised distances and color excesses in order to provide homogeneous estimates for all groups with uvby $\beta$ photometry in the field under consideration.

NGC 3293 is a bright open cluster embedded in an emission nebula. Shobbrook (1980) obtained uvby $\beta$ photometry of a significant amount of cluster members and calculated true $\mathrm{DM}=12.75(3.55 \mathrm{kpc})$. This estimate however has been based on a preliminary $M_{V}-\beta$ calibration, later found to overestimate the brightness, and was corrected to $11.95 \pm 0.1 \mathrm{mag}$ (2455 pc) (Shobbrook 1983). Our estimate is based on 61 stars with available uvby $\beta$ photometry and yields a true (median) $\mathrm{DM}=12.15$ ( $\pm 0.43 \mathrm{sd} ; \pm 0.05 \mathrm{se}$ ), corresponding to a distance of 2691 pc. A distance of 2373 pc is provided in the Dias et al. (2003) catalog, while Kharchenko et al. (2005) estimate 2471 pc. Although a fair agreement exists between our estimate and the most recently published distances, our result locates the cluster some $250 \mathrm{pc}$ farther than currently accepted. The stars are uniformly reddened with average color excess $E(b-y)=0.20( \pm 0.05 \mathrm{sd} ; \pm 0.01 \mathrm{se})$.

NGC 3114 has been observed in the uvby $\beta$ system by Schmidt (1982) and Schneider \& Weiss (1988). In this cluster, there are 29 stars earlier than B9 with uvby $\beta$ data presently available. The cluster is located in a crowded low-reddened field, thus complicating the separation of cluster members from field stars. Carraro \& Patat (2001) obtained UBVRI photometry of more than 2000 stars near the center of the cluster. They found this region to 
be heavily contaminated by field stars and separated two populations: several low reddened stars which are presumably cluster members, and field stars having larger reddening. All stars in our sample are low reddened. We estimate an average color excess $E(b-y)=$ 0.053 ( $\pm 0.01 \mathrm{sd} ; \pm 0.009 \mathrm{se})$, in agreement with Schmidt (1982) and Carraro \& Patat (2001). However, these stars show a significant spread in distance and not well defined MS on the $V_{0}$ vs. $(b-y)_{0}$ diagram (Fig. 2). After excluding one star with very large distance, the remaining 28 stars yield a median true $\mathrm{DM}=10.06( \pm 0.71 \mathrm{sd} ; \pm 0.13 \mathrm{se})$, corresponding to 1028 pc (vs. 911 pc listed by Dias et al. (2003) and 1130 pc provided by Schmidt (1982)). Excluding the stars to the left of MS does not significantly affect this distance estimate. Nineteen stars, however, appear to be nicely grouped between distance moduli 9.7 and 10.5 mag, at an average true $\mathrm{DM}=10.01( \pm 0.29 \mathrm{sd} ; \pm 0.067 \mathrm{se})$. Thus, we provide a revised distance of $1005 \pm 31$ pc to NGC 3114, which is in fair agreement with the estimate of Carraro $\&$ Patat (2001) of $920 \pm 50$ pc and Schneider \& Weiss (1988) of $940 \pm 60$ pc.

\subsection{Westerlund 2}

The massive, young stellar cluster Wd $2\left(l=284.2^{\circ}, b=-0.33^{\circ}\right)$ is considered to be one of the five superclusters known in the Milky Way. The cluster is thought to be spatially connected to the HiI complex RCW 49, a remarkable infrared nebula as revealed by Spitzer (Churchwell et al. 2004), and perhaps to the extended TeV $\gamma$-ray source HESS J1023-575 (Aharonian et al. 2007). Recently, $\gamma$-rays in the $\mathrm{MeV} / \mathrm{GeV}$ energy domain have been reported from the same direction by the Fermi collaboration (Abdo et al. 2009). Fujita et al. (2009) present an analysis of the diffuse X-ray emission of Wd 2 which may indicate a recent $\left(\sim 10^{5}-10^{6}\right.$ yrs ago) explosion of a massive star. Recently a new hard spectrum TeV $\gamma$-ray source, HESS J1026582, was discovered by the H.E.S.S. collaboration Abramowski et al. 2011). Ackermann et al. (2011) showed that this emission is due to a $\gamma$-ray pulsar with a preferred distance of $2.4 \mathrm{kpc}$. Since the most recent distance estimates to $\mathrm{Wd} 2$ were established to be in the $5-8 \mathrm{kpc}$ range, this $\gamma$-ray emission might be unrelated to $\mathrm{Wd} 2$ and this would cast serious doubt on a connection between the pulsar and the cluster (see Rauw et al. (2011) for a thorough discussion). Wd 2 is one of the clusters in the Galaxy for which associated molecular clouds have been identified (Furukawa et al. 2009, see also Dame 2007). Fukui et al. (2009) discovered a spectacular jet and arc of molecular gas detected with the NANTEN telescope in the ${ }^{12} \mathrm{CO} J=1-0115 \mathrm{MHz}$ emission line survey (Muzino \& Fukui 2004).

The total stellar mass in Wd 2 is of the order of $4500 M_{\odot}$, including $12 \mathrm{O}$ stars and

2 WR stars (Rauw et al. 2007). Due to the high (and apparently local) extinction, most 
of the photometric and spectroscopic studies are restricted to the brightest stars. The only deep BVI CCD photometry is presented by Carraro \& Munari (2004). The distance to Wd 2 has been a very controversial issue (see Dame (2007) and the references therein) and varies between 2 and $8.3 \mathrm{kpc}$.

Distances to RCW 49 have been presented by several authors (see also 4.1). In the study of Grabelsky et al. (1988) Wd 2 and RCW 49 are associated with GMC 7, at an optical distance of $4 \mathrm{kpc}$. Russeil (2003) determine a kinematic distance of $4.7(+0.6,-0.2)$ $\mathrm{kpc}$ to RCW 49. On the basis of an analysis of the $\mathrm{CO}$ emission and $21 \mathrm{~cm}$ absorption along the line of sight to Wd 2, Dame (2007) argued that Wd 2 must be associated with GMC 8 of the study of Grabelsky et al. (1988), in the far side of the Carina arm. Dame (2007) determined a kinematic distance of $6.0 \pm 1.0 \mathrm{kpc}$ to the molecular structure toward $\mathrm{Wd} 2$, while Furukawa et al. (2009) obtained $5.5 \pm 1.5 \mathrm{kpc}$. In their original study, Grabelsky et al. (1988) reported a kinematic distance of $6.6 \mathrm{kpc}$ to GMC 8.

Although uvby $\beta$ photometry of the stars in $\mathrm{Wd} 2$ is not available, we attempt here a distance estimate for this cluster. The 12 O-type stars, studied by Rauw et al. (2007) are listed in Table 3. Similarly to Rauw et al. we use the MK type to obtain $M_{V}$ and $E(B-V)$, but utilizing the calibration by Deutschman et al. (1976). We have tested this calibration based on a large sample of O-B stars and found it to provide $M_{V}$ that is in agreement with the uvby $\beta$ photometry. For example, the uvby $\beta$ sample used in this paper contains six Otype stars. For them, the average $M_{V}$ derived by uvby $\beta$ photometry is $-5.2 \pm 0.26 \mathrm{mag}$, and the average spectroscopic $M_{V}$ is $-5.08 \pm 0.13$ mag. To do another check we recalculated the distance moduli of the stars of the Lodén group with available spectral classification and found a median value of $11.19( \pm 1.46 \mathrm{sd} ; \pm 0.55 \mathrm{se})$, which, despite of the somehow larger spread, is in agreement with the uvby $\beta$ estimate.

The $E(B-V)$ and $M_{V}$ obtained here are listed in columns 7 and 8 of Table 3 . Columns 9, 10 and 11 contain the distance moduli calculated for three values of $\mathrm{R}(3.2,4.2,5.2)$. The average distance moduli are shown at the bottom of Table 3, together with the mean error, and correspond to distances of $6700 \mathrm{pc}, 3090 \mathrm{pc}$ and $1432 \mathrm{pc}$, respectively. Since the $A_{V}$ toward Wd 2 is more than $5 \mathrm{mag}$, one could expect an abnormal value of $\mathrm{R}$, but, as mentioned by Rauw et al. (2007), the present photometric data does not allow us to evaluate the reddening law toward $\mathrm{Wd} 2$. Another way to test our distance estimate is to obtain $(b-y)_{0}$ from $(B-V)_{0}$ and plot the Wd 2 stars on the $M_{V}$ vs $(b-y)_{0}$ diagram. To do this the study of Crawford (1975b, his Fig. 7) on the O-type stars was used. The average $(b-y)_{0}$ and $M_{V}$ values for the 12 stars are -0.14 and -5.5 mag (obtained via the calibration of Deutschman et al. (1976)), respectively, providing a good agreement with the location of the MS on Fig. 2, where the $M_{V}$ values are obtained via the calibration 
of Balona \& Shobbrook (1984). This points out that both $M_{V}$ and $(B-V)_{0}$ obtained from the Deutschman et al. (1976) calibration for the Wd 2 stars are in agreement with the parameters provided by the Strömgren photometry. Thus, utilizing $\mathrm{R}=3.2$, we provide a new photometric distance to $\mathrm{Wd} 2$ of $6698_{-475}^{+512} \mathrm{pc}$.

The accurate and deep BVI CCD photometry by Carraro \& Munari (2004) provides a distance of $6.4 \pm 0.4 \mathrm{kpc}$, also in excellent agreement with the one of $6.7 \mathrm{kpc}$ derived here. However, an investigation of the value of $\mathrm{R}$ is clearly warranted in order to pinpoint the exact distance to the cluster.

In their study, Rauw et al. have used the new calibration for O-type stars by Martins \& Plez (2006). In order to closely evaluate both calibrations, we used the database of Maíz Apellániz et al. (2004) and calculated color excess and absolute magnitude for all 273 stars with uvby $\beta$ photometry. Then we obtained these parameters via the calibrations of Martins \& Plez (2006) and Deutschman et al. (1976). The comparisons led to the following results. For LC V (96 stars) Deutschman et al. (1976) provides DM practically identical with the uvby $\beta$ ones, while Martins \& Plez (2006) seem to slightly underestimate the DM by $0.25 \pm 0.47$. For LC III (44 stars) Martins \& Plez (2006) provides DM practically identical with the uvby $\beta$ ones, while Deutschman et al. (1976) seem to underestimate the DM by $0.29 \pm 0.73$. In the $\mathrm{Wd} 2$ sample, seven of the stars are of LC V and 5 stars are LC III. The calibration of Deutschman et al. (1976) provides DM $=14.095 \pm 0.43$ for the $7 \mathrm{LC} \mathrm{V}$ stars and $\mathrm{DM}=14.18 \pm 0.77$ for the $5 \mathrm{LC}$ III stars. For the same sample the calibration of Martins \& Plez (2006) provides DM=14.08 \pm 0.53 for LC V and DM=14.72 \pm 0.79 for LC III. It seems that in this particular case the Deutschman et al. (1976) calibration provides more consistency between LC V and LC III. Overall this is an acceptable difference in the average DM which yields to $6.7 \mathrm{kpc}$ using Deutschman et al. (1976) and $7.4 \mathrm{kpc}$ if Martins \& Plez 【(2006) is utilized. All of the above calculations are for $\mathrm{R}=3.2$. In their study Rauw et al. (2007) use $\mathrm{R}=3.1$, which, since the reddening of $\mathrm{Wd} 2$ is quite high, results in a distance of $8 \mathrm{kpc}$. All this points out that our result is actually consistent with that of Rauw et al. (2007) and the discrepancy is mostly due to the utilized value of R.

Figure 7 presents the plots of DM vs. Galactic longitude and Galactic latitude for all stars of this sample with calculated distances. The clusters and cluster candidates are shown, together with the GMC from the study of Grabelsky et al. (1988). For the clusters studied here we use the new estimates and utilize the distances listed in Table 2 for the rest of the clusters. The edge of the Carina arm based on the distribution of the bright OB stars Graham 1970; Kaltcheva \& Scorcio 2010) is indicated with a solid line. The distances to GMC 3 and GMC 7 are optical, all other GMC distances are kinematic (see Grabelsky et al. (1988)). Any distance larger than $3.5 \mathrm{kpc}$ would place Wd 2 beyond the edge of the Carina 
arm, but a large distance is in agreement with the location of the edge of the arm delineated by the GMC. There is an excellent agreement between the newly obtained distance of 6.7 $\mathrm{kpc}$ to Wd 2 and the kinematic distance of GMC 8.

\section{Conclusions}

We present a uvby $\beta$ photometric investigation of a number of clusters and cluster candidates and field stars located in the Galactic plane toward the tangent of the Carina arm. Based on the derived homogeneous distances and color excesses of more than 260 stars of spectral types $\mathrm{O}$ to $\mathrm{G}$, we provide revised distances for the stellar groups and layers present in this sample. The main findings are as follows:

1. The cluster candidate Lodén 112 seems to be a physical group at $1629_{-80}^{+84}$ pc. We found other $\mathrm{OB}$ stars with similar proper motions at that same distance, and suggest a new $\mathrm{OB}$ association at coordinates $282^{\circ}<l<285^{\circ},-2^{\circ}<b<2^{\circ}$. This feature appears connected to Lodén 112 and containing at least the two brightest OB stars of IC 2581. It is located at $1682_{-104}^{+113} \mathrm{pc}$ and probably related to the HiI nebulosities seen in this direction.

2. The following parameters are obtained for the luminous blue variable HR Car: $M_{V}$ $=-8.3, A_{V}=3.96 \mathrm{mag}$ (for $R=3.2$ ) and a distance of $3 \mathrm{kpc}$ (adopting a visual magnitude of 8.076). The currently accepted distance of $5 \mathrm{kpc}$ to this star seems overestimated.

3. The high interstellar extinction toward CPD $-553036\left(A_{V}=5.36 \mathrm{mag}\right)$ and its proximity to RCW 45 in terms of Galactic coordinates may indicate a possible relation to the nebula. The star is located at 1101 pc photometric distance.

4. The distance of $542_{-105}^{+131}$ pc to the open cluster Lodén 46 is in a good agreement with the currently accepted value. However, one can notice that the photometric distances obtained here show somehow a spread larger than expected for a nearby cluster. This does not necessary mean a doubtful nature for this cluster and can be due to the fact that the stars in Lodén 46 are of spectral types A0-A2 and the uvby $\beta$ system may not provide accurate stellar parameters for this spectral range.

5. Based on 61 B-type stars with available uvby $\beta$ photometry in NGC 3293 we find a distance of 2691 pc. Although a fair agreement exists between our estimate and the most recently published distances, our result locates this cluster some 250 pc farther than currently accepted.

6. We provide a revised distance of $1005 \pm 31$ pc to the open cluster NGC 3114 . 
7. Utilizing BV photometry and spectral classification of the known O-type stars in $\mathrm{Wd} 2$, we provide a new distance estimate of $6698_{-475}^{+512} \mathrm{pc}$, in excellent agreement with recent distance determination to the giant molecular structures in this direction. This estimate does not contradict with the 8-kpc distance provided by Rauw et al. (2007) as the difference is due to a large extent to the utilized value of $R$.

This work is supported by the National Science Foundation grant AST-0708950 and an University of Wisconsin Oshkosh faculty development award. N.K. acknowledges support from the SNC Endowed Professorship at the University of Wisconsin Oshkosh. V.G. acknowledges support by the Bulgarian National Science Research Fund under the grants DO 02-85/2008 and DO 02-362/2008. This research has made use of the SIMBAD database, operated at CDS, Strasbourg, France. We acknowledge the use of NASA's SkyView facility (http://skyview.gsfc.nasa.gov) located at NASA Goddard Space Flight Center (see McGlynn et al. 1998). SkyView is a Virtual Observatory on the Net generating images of any part of the sky at wavelengths in all regimes from Radio to Gamma-Ray. We acknowledge the use of the Southern H-Alpha Sky Survey Atlas (SHASSA), which is supported by the National Science Foundation (Gaustad et al. 2001). We are thankful to an anonymous referee for many valuable comments that significantly improved the paper.

\section{REFERENCES}

Abdo, A. A., Ackermann, M., Ajello, M., Atwood, W. B., Axelsson, M., Baldini, L., Ballet, J., Band, D. L., Barbiellini, G., Bastieri, D., et al. 2009, ApJS, 183, 46

Abramowski, A., Acero, F., Aharonian, F., Akhperjanian, A. G., Anton, G., Barnacka, B., Barres de Almeida, U., et al. 2011, A\&A, 525A, 46

Ackermann, M., Ajello, M., Baldini, L.; Ballet, J., Barbiellini, G., et al. 2011, ApJ, 726, 35

Aharonian, F., Akhperjanian, A. G., Bazer-Bachi, A. R., Beilicke, M., Benbow, W., Berge, D., Bernlöhr, K., et al. 2007, A\&A, 467, 1075

Ascenso, J., Alves, J., Beletsky, Y, \& Lago, M.T.V.T. 2007, A\&A, 466, 137

Balona, L. A. 1994, MNRAS, 268, 119

Balona, L. A., \& Shobbrook, R. R. 1984, MNRAS, 210, 375

Bloemen, J. B., Deul, E. R., \& Thaddeus, P. 1990, A\&A, 233, 437 
Bronfman, L. 1992, in The center, bulge and disk of the Milky Way, ed. L. Blitz, p. 131

Carlson, E., D., \& Henize, K., G. 1979, Vistas Astron, 23, 213

Carraro, G., \& Munari, U. 2004, MNRAS, 347, 625

Carraro, G., \& Patat, F. 2001, A\&A, 379, 136

Cichowolski, S., Romero, G. A., Ortega, M. E., Cappa, C. E., \& Vasquez, J. 2009, MNRAS, 394,900

Crawford, D. L. 1975a, AJ, 80, 955

Crawford, D. L. 1975b, PASP, 87, 481

Crawford, D. L. 1978, AJ, 83, 48

Crawford, D. L. 1979, AJ, 84, 1858

Churchwell, E., Whitney, B. A., Babler, B. L., Indebetouw, R., Meade, M. R., Watson, Ch., Wolff, M. J., Wolfire, M. G., et al. 2004, ApJS, 154, 322

Dame, T. M., Hartmann, D., \& Thaddeus, P. 2001, ApJ, 547, 792

Dame, T. M. 2007, ApJ, 665L, 163

Denoyelle, J. 1977, A\&AS, 27, 343

Dias, W. S., Alessi, B. S., Moitinho, A., \& Lépine, J. R. D. 2003, EAS Publ. Series, Vol. 10, p. 195

Deutschman, W. A., Davis, R. J., \& Schild, R. E. 1976, ApJS, 30, 97

Duncan, A. R., Haynes, R. F., Stewart, R. T., \& Jones, K. L. 1995, MNRAS, 277, 319

Gaustad, J. E., McCullough, P. R., Rosing, W., \& Van Buren D. 2001, PASP, 113, 1326

Grabelsky, D. A., Cohen, R. S., Bronfman, L., Thaddeus, P., \& May, J. 1987, ApJ, 315, 122

Grabelsky, D. A., Cohen, R. S., Bronfman, L., \& Thaddeus, P. 1988, ApJ, 331, 181

Graham, J.A. 1970, AJ, 75, 703

Finkbeiner, D. P. 2003, ApJS, 146, 407

Fujita, Y., Hayashida, K., Takahashi, H., \& Takahara, F. 2009 PASJ, 61, 1229 
Fukui, Y., Furukawa, N., Dame, T. M., Dawson, J. R., Yamamoto, H., Rowell, G. P., Aharonian, F. Hofmann, W., de Oña-Wilhelmi, E., Minamidani, T., Kawamura, A., Mizuno, N., Onishi, T., Mizuno, A., \& Nagataki, S. 2009, PASJ, 61L, 23

Furukawa, N., Dawson, J. R., Ohama, A., Kawamura, A., Mizuno, N., Onishi, T., \& Fukui, Y. 2009, ApJ, 696L, 115

Hauck, B., \& Mermilliod, M. 1998, A\&AS, 129, 143

Hilditch, R. W., Hill, G., \& Barnes, J. V. 1983, MNRAS, 204, 241

Humphreys, R. M. 1978, ApJS, 308, 309

Hutsemékers, D., \& van Drom, E. 1991, A\&A, 248, 141

Kaltcheva, N. 2003, A\&A, 410, 523

Kaltcheva, N.T., Olsen, E.H., \& Clausen, J.-V., 2000, A\&AS, 146, 365

Kaltcheva, N., \& Golev, V. 2011, eprint arXiv:1107.3758

Kaltcheva, N., \& Hilditch, R. 2000, MNRAS, 312, 753

Kaltcheva, N., \& Makarov, V. 2007, ApJ, 667L, 155

Kaltcheva, N., \& Scorcio, M. 2010, A\&A, 514, A59

Kharchenko, N.V., Piskunov, A.E., Röser, S., Schilbach, E., \& Scholz, R.-D. 2005, A\&A, 438, 1163

Kilkenny, D. 1993, South Afr. Astron. Obs., Circ. No. 15, p. 53

Kilkenny, D., \& Whittet, D. C. B. 1985, MNRAS, 216, 127

Kilkenny, D., \& Whittet, D. C. B. 1993, South Afr. Astron. Obs., Circ. No. 15, p. 40

Koo, B.-C., Heiles, C., \& Reach, W. T. 1992, ApJ, 390, 108

Lloyd-Evans, T. 1969, MNRAS, 146, 101

Lodén, L. O. 1977, A\&AS, 29, 31

Lodén, L. O. 1979, A\&AS, 36, 83

Maíz Apellániz, J. Walborn, N. R., Galu, H. A., \& Wei, L. H. 2004, ApJS, 151, 103 
Martins, F., \& Plez, B. 2006, A\&A, 457, 637

McClure-Griffiths, N. M., Dickey, J. M., Gaensler, B. M., Green, A. J., Haynes, R. F., \& Wieringa, M. H. 2000, AJ, 119, 2828

McGlynn, T., Scollick, K., \& White, N. 1998, in New Horizons from Multi-Wavelength Sky Surveys, Proc. of the 179th Symp. of the IAU, held in Baltimore, USA Aug 26-30, 1996, Kluwer Academic Publishers, edited by B. J. McLean, D. A. Golombek, J. J. E. Hayes, \& H. E. Payne, p. 465

Melnik, A. M., \& Efremov, Yu. N. 1995, AstL, 21, 10

Moffat, A. F. J., \& Shara, M. M., \& Potter, M. 1991, AJ, 102, 642

Mizuno, A., \& Fukui, Y. 2004, in Milky Way Surveys: The Structure and Evolution of our Galaxy, ed. D. Clemens, R. Shah, \& T. Brainerd, ASP Conf. Ser., 317, 59

Rauw, G., Manfroid, J., Gosset, E., Nazé, Y., Sana, H., De Becker, M., Foellmi, C., \& Moffat, A. F. J. 2007, A\&A, 463, 981

Rauw, G., Sana, H., \& Nazé, Y. 2011, A\&A, 535, A40

Reed, B. C. 2003, AJ, 125, 2531

Russeil, D. 2003, A\&A, 397, 133

Parthasarathy, M., Jain, S. K., \& Bhatt, H. C. 2000, A\&A, 355, 221

Schlegel, D. J., Finkbeiner, D. P., \& Davis, N. 1998, ApJ, 500, 525

Schneider H., \& Weiss, W. W. 1988, A\&AS, 75, 353

Schmidt, E. G. 1982, PASP, 94, 232

Shobbrook, R. R. 1980, MNRAS, 92, 821

Shobbrook, R. R. 1983, MNRAS, 205, 1251

Strömgren , B. 1966, ARA\&A, 4, 433

Taylor, J. H., \& Cordes, J. M. 1993, ApJ, 411, 674

Tsujimoto, M., Feigelson, E. D., Townsley, L. K., Broos, P. S., Getman, K. V., Wang, J., Garmire, G. P., Baba, D., Nagayama, T., Tamura, M., \& Churchwell, E. B., 2007, ApJ, 665, 719 
Turner, D. G. 1973, AJ, 78, 597

Umana, G., Buemi, C. S., Trigilio, C., Hora, J., L., Fazio, G. G., \& Leto, P. 2009, ApJ, 694, 697 J. P. 2002, ApJ, 566, 261

Vallée, J. P. 2008, ApJ, 135, 1301

van Genderen, A., M., Robijn, F. H. A., van Esch, B. P. M., \& Lamers, H.J.G.L.M., 1991, A\&A, 246, 407

van Leeuwen, F. 2007, Hipparcos, the new reduction of the raw data (Dordrecht:Springer)

Viotti, R. 1971, PASP, 83, 170

Wramdemark, S. 1980, A\&AS, 41, 33 

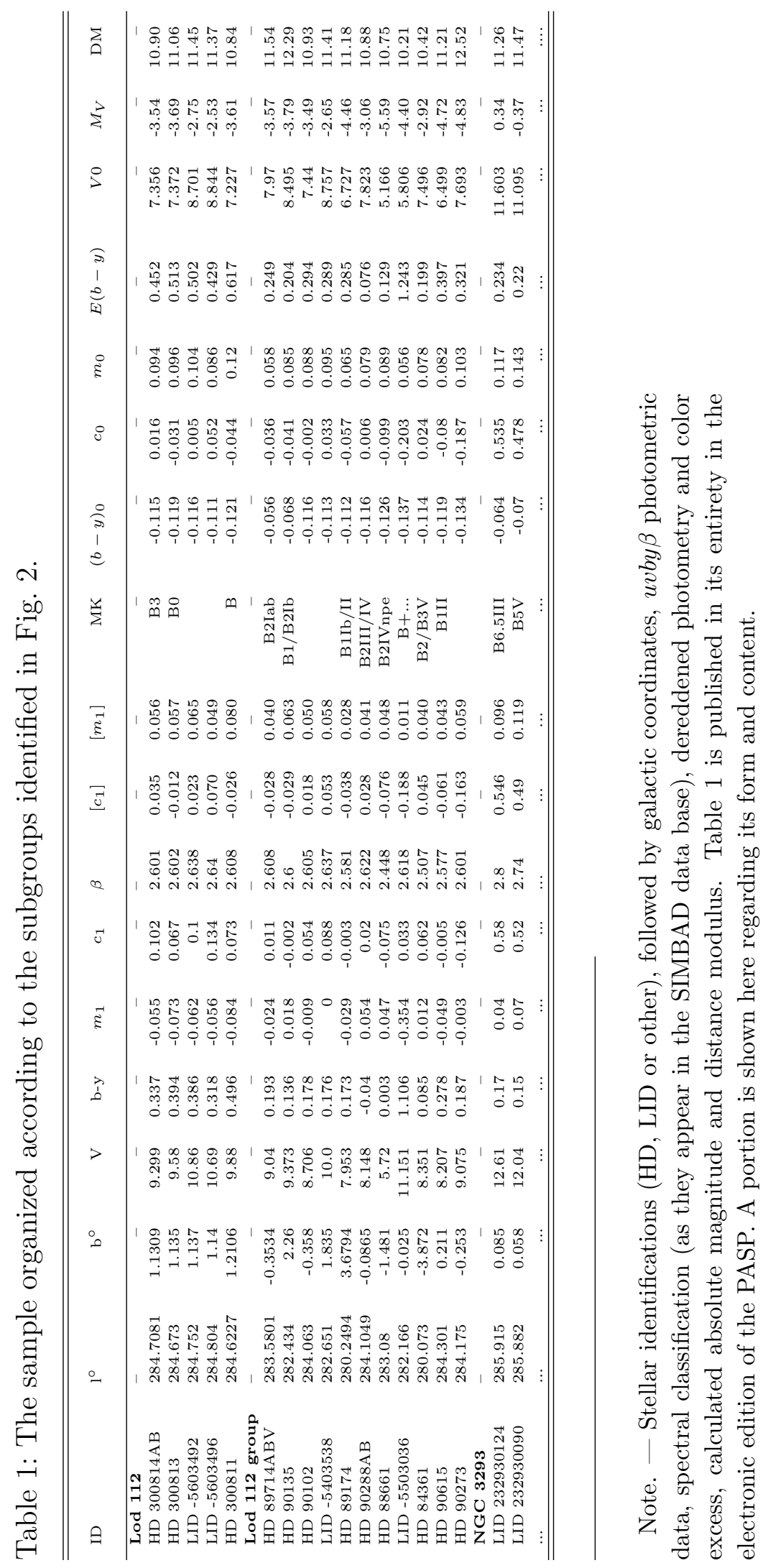
Table 2: Known clusters (Dias et al. 2003) and Lodén cluster candidates in the field studied.

\begin{tabular}{|c|c|c|c|c|c|}
\hline Name & $\begin{array}{l}l \\
\text { deg }\end{array}$ & $\begin{array}{l}b \\
\text { deg }\end{array}$ & $\begin{array}{r}\text { Distance } \\
\mathrm{pc} \\
\end{array}$ & $\begin{array}{l}\text { Age } \\
\text { Myr }\end{array}$ & $\begin{array}{l}\mathrm{DM} \\
\mathrm{mag}\end{array}$ \\
\hline H 90 & 283.099 & -1.476 & 2572 & 7.943 & 12.05 \\
\hline NGC 3114 & 283.332 & -3.84 & 911 & 8.093 & 9.79 \\
\hline NGC 3330 & 284.188 & 3.848 & 894 & 8.229 & 9.75 \\
\hline Westerlund 2 & 284.276 & -0.336 & 6400 & 6.3 & 14.03 \\
\hline Collinder 220 & 284.567 & -0.342 & 1547 & 8.083 & 10.94 \\
\hline IC 2581 & 284.588 & 0.035 & 2446 & 7.142 & 11.94 \\
\hline Saurer 3 & 285.096 & 2.999 & 9550 & 9.3 & 14.90 \\
\hline NGC 3293 & 285.856 & 0.074 & 2327 & 7.014 & 11.83 \\
\hline NGC 3255 & 286.088 & -2.635 & 1445 & 8.3 & 10.79 \\
\hline NGC 3324 & 286.228 & -0.188 & 2317 & 6.754 & 11.82 \\
\hline Carraro 1 & 286.236 & -0.29 & 1900 & 9.48 & 11.39 \\
\hline Collinder 223 & 286.358 & -1.705 & 2820 & 8 & 12.25 \\
\hline Lodén 1 & 281.02 & -0.17 & 360 & 9.29 & 7.78 \\
\hline Lodén 27 & 282.171 & -0.335 & & & \\
\hline Lodén 28 & 282.21 & -2.21 & 3950 & 7.3 & 12.98 \\
\hline Lodén 46 & 282.6 & 2.07 & 540 & 9.03 & 8.66 \\
\hline Lodén 59 & 283.08 & 3.28 & 650 & 8.45 & 9.06 \\
\hline Lodén 89 & 284.35 & 0.87 & 380 & 8.47 & 7.89 \\
\hline Lodén 112 & 284.68 & 1.17 & 2500 & 6.96 & 11.98 \\
\hline Lodén 143 & 285.3 & -0.89 & 600 & 8.45 & 8.89 \\
\hline Lodén 153 & 285.67 & 0.09 & 2670 & 6.74 & 12.13 \\
\hline Lodén 165 & 285.935 & -0.365 & 1900 & 9.48 & 11.39 \\
\hline Lodén 172 & 286.285 & -2.502 & & & \\
\hline Lodén 189 & 286.72 & 2.59 & 720 & 8.64 & 9.28 \\
\hline
\end{tabular}

Note. - The object's name and galactic coordinates are given in the first three columns, followed by distance and age (where available), and true distance moduli. All data in this table are adopted from the catalog of Dias et al. (2003).

Table 3: Distance and color excess for the O-type stars in Westerlund 2.

\begin{tabular}{|c|c|c|c|c|c|c|c|c|c|c|}
\hline MSP No & MK & $B$ & $V$ & $B-V$ & $(B-V)_{0}$ & $M_{V}$ & $E(B-V)$ & $\mathrm{DM}(\mathrm{R}=5.2)$ & $\mathrm{DM}(\mathrm{R}=4.2)$ & $\mathrm{DM}(\mathrm{R}=3.2)$ \\
\hline 18 & $\mathrm{O} 5 \mathrm{~V}$ & 14 & 12.8 & 1.2 & -0.34 & -5.6 & 1.54 & 10.39 & 11.93 & 13.47 \\
\hline 151 & O6III & 15.6 & 14.33 & 1.27 & -0.332 & -5.5 & 1.60 & 11.50 & 13.10 & 14.70 \\
\hline 157 & $06.5 \mathrm{~V}$ & 15.5 & 14.14 & 1.36 & -0.332 & -5.3 & 1.69 & 10.64 & 12.33 & 14.03 \\
\hline 167 & O6III & 15.48 & 14.19 & 1.29 & -0.332 & -5.5 & 1.62 & 11.26 & 12.88 & 14.50 \\
\hline 171 & $\mathrm{O} 5 \mathrm{~V}$ & 15.83 & 14.44 & 1.39 & -0.34 & -5.6 & 1.73 & 11.04 & 12.77 & 14.50 \\
\hline 175 & $\mathrm{O} 6 \mathrm{~V}$ & 15.15 & 13.93 & 1.22 & -0.335 & -5.4 & 1.55 & 11.24 & 12.80 & 14.35 \\
\hline 182 & O4III & 15.69 & 14.43 & 1.26 & -0.34 & -5.6 & 1.60 & 11.71 & 13.31 & 14.91 \\
\hline 183 & O3V & 15.03 & 13.61 & 1.42 & -0.34 & -5.6 & 1.76 & 10.06 & 11.82 & 13.59 \\
\hline 188 & O4III & 14.6 & 13.32 & 1.28 & -0.34 & -5.6 & 1.62 & 10.50 & 12.12 & 13.74 \\
\hline 199 & O3V & 15.74 & 14.39 & 1.35 & -0.34 & -5.6 & 1.69 & 11.20 & 12.89 & 14.58 \\
\hline 203 & O6III & 14.66 & 13.22 & 1.44 & -0.332 & -5.5 & 1.77 & 9.51 & 11.28 & 13.05 \\
\hline \multirow[t]{3}{*}{263} & O6V & 16.5 & 14.91 & 1.59 & -0.335 & -5.4 & 1.92 & 10.30 & 12.22 & 14.15 \\
\hline & & & & & & & & 10.78 & 12.45 & 14.13 \\
\hline & & & & & & & & \pm 0.19 s.e. & \pm 0.17 s.e. & \pm 0.16 s.e. \\
\hline
\end{tabular}

Note. - MSP identification (Moffat et al. 1991), MK classification and $B$ and $V$ magnitudes from SIMBAD. $(B-V)_{0}$ and $M_{V}$ are based on the calibration of Deutschman et al. (1976). The last three columns present the distance moduli calculated for $\mathrm{R}=5.2,4.2$ and 3.2. The average distance moduli and the mean errors are shown at the end of the table for the three values of $\mathrm{R}$. 


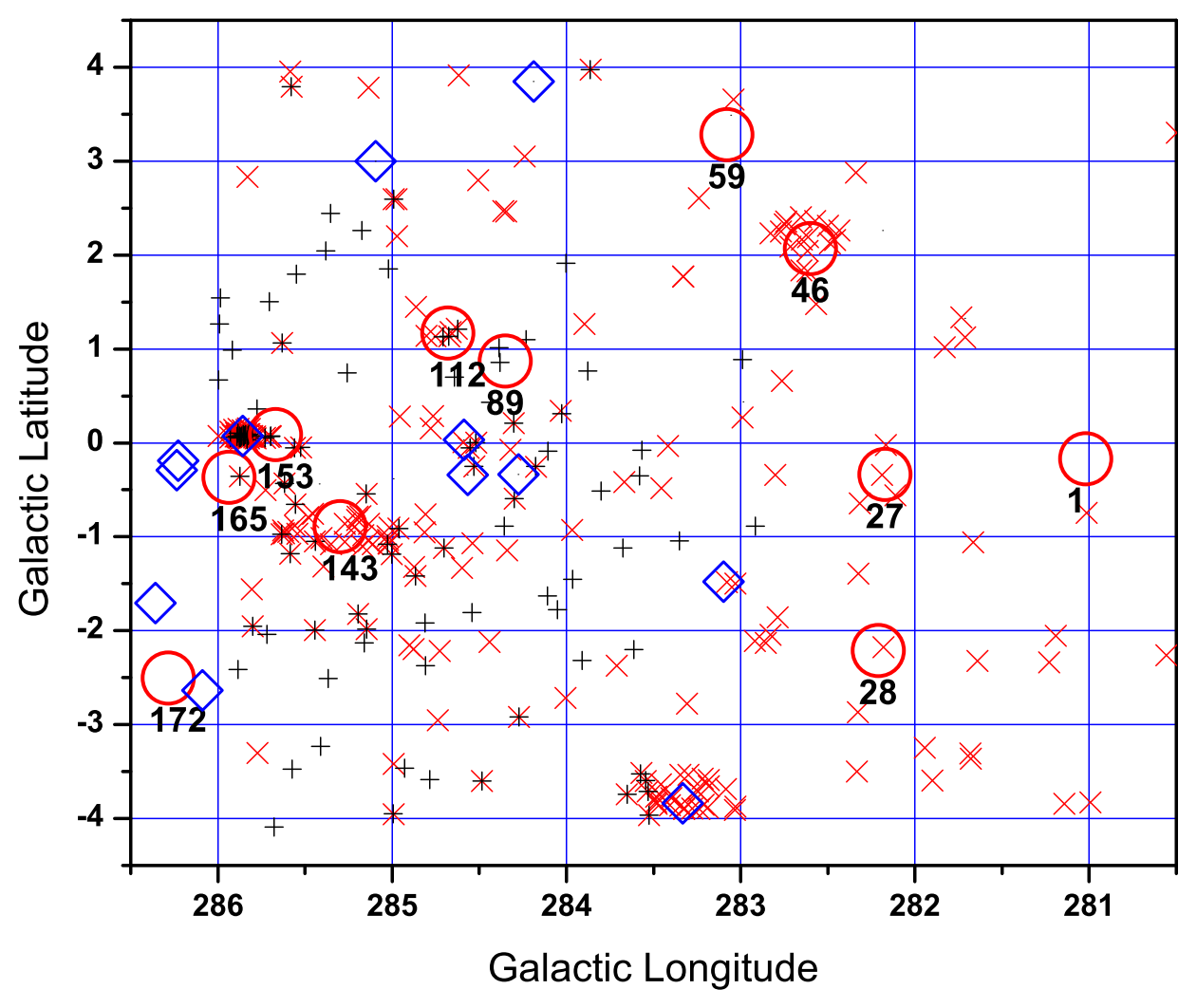



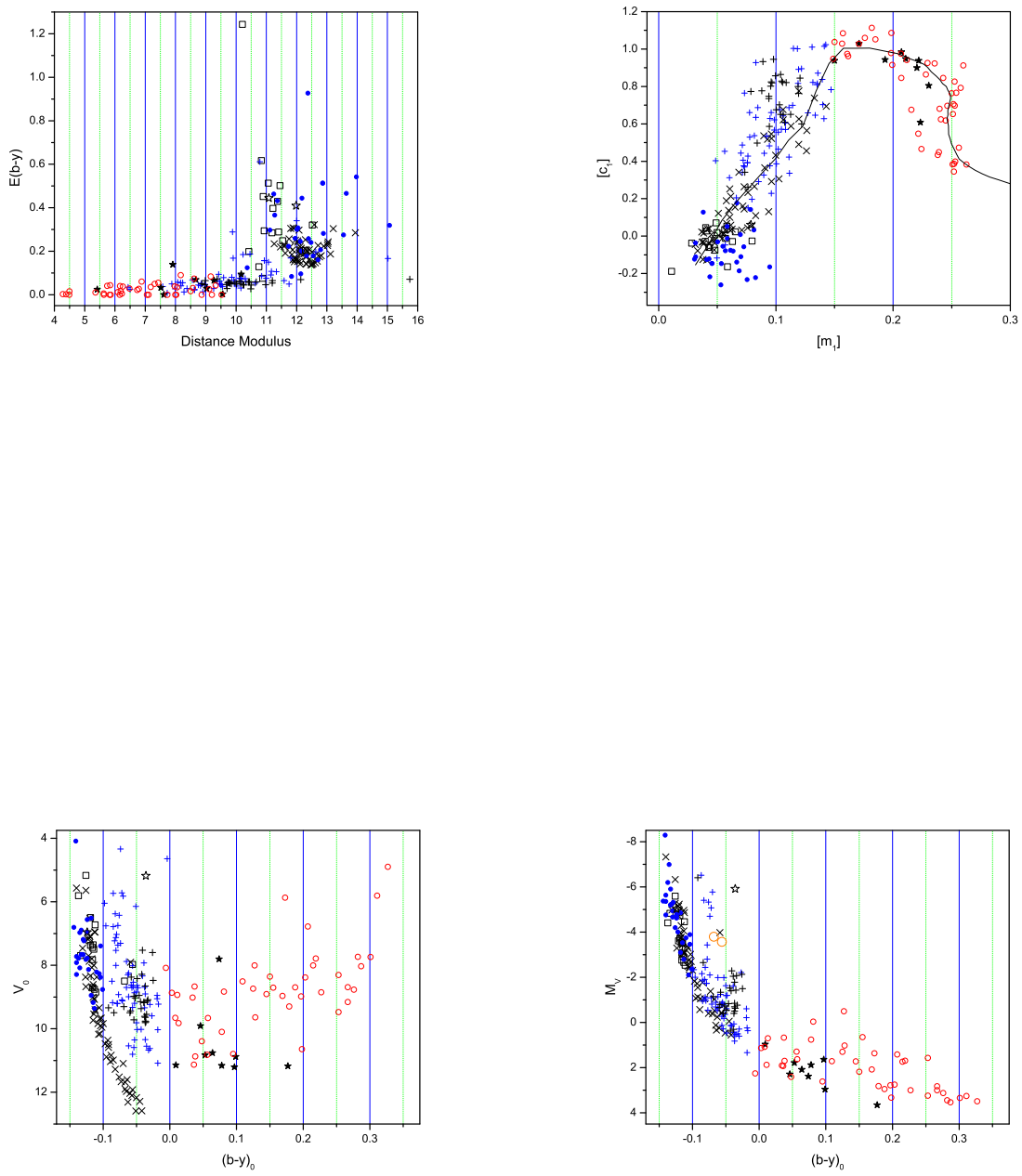

Fig. 2.- All stars with calculated distances and color excesses (see Table 1). The sample is separated into subgroups as follows: Lodén 112 group (11 stars) and Lodén 112 cluster (5 stars): open squares; NGC 3293 (62 stars): x symbols; NGC 3114 (29 stars): thick + symbols; IC 2581 (2 stars): open-star symbols; Lodén 46 (9 stars): filled star symbols; blue MS field stars (29): filled circles; blue evolved field stars (66): + symbols; field stars of A-F-G types (43): open circles. See the electronic edition of the PASP for a color version of this figure. 


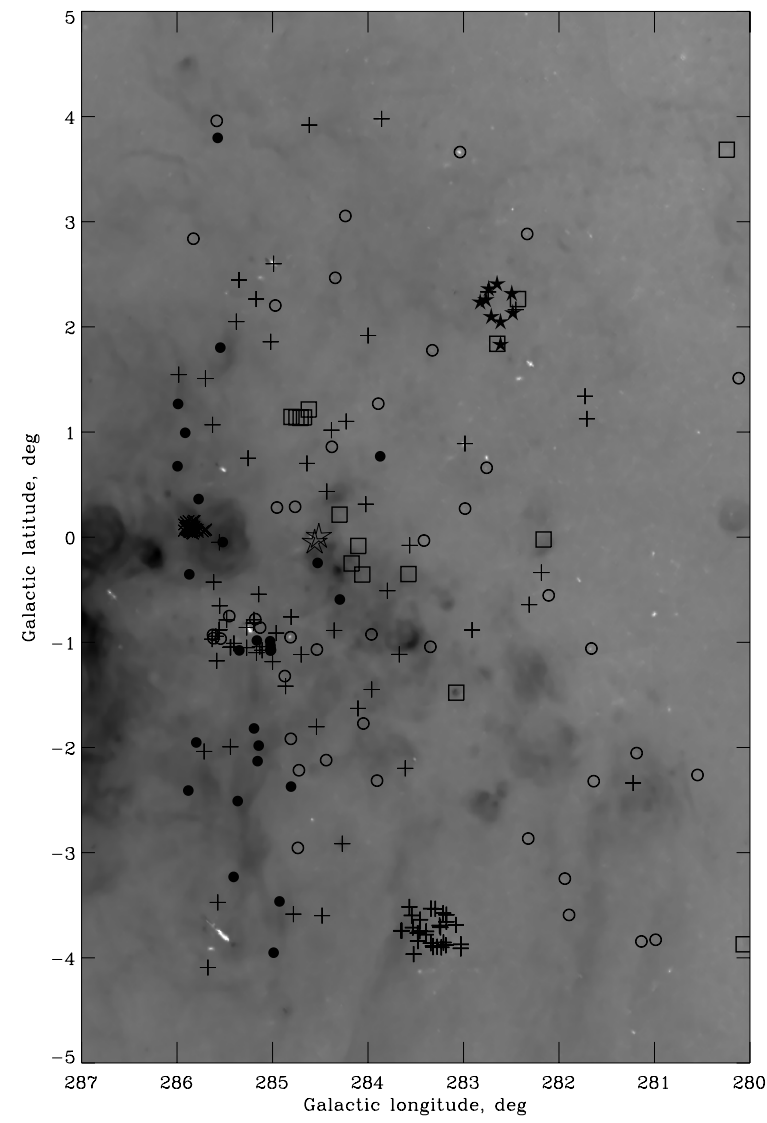

Fig. 3.- All stars with calculated distances and color excesses overplotted on $\mathrm{H} \alpha$ map (smoothed to 4 arcmin resolution to remove star residuals, Gaustad et al. (2001)), obtained via the SkyView VO interface (see McGlynn et al. 1998). The white spots and patches in the $\mathrm{H} \alpha$ image are artefacts after star's subtraction. The symbols are the same as in Fig. 2. 


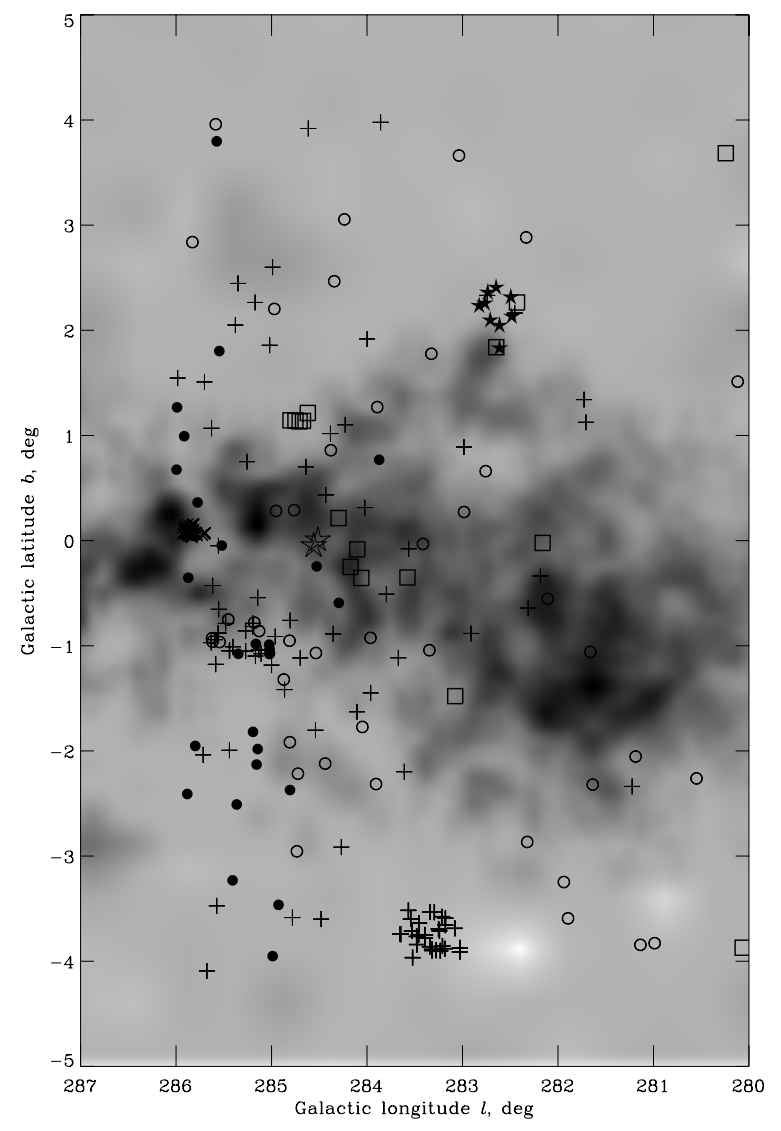

Fig. 4.- All stars with calculated distances and color excesses overplotted on ${ }^{12} \mathrm{CO}(\mathrm{J}=1 \rightarrow 0$, $115 \mathrm{GHz}$ ) map of the region (Dame et al. 2001), obtained via the SkyView VO interface (see McGlynn et al. 1998). The symbols are the same as in Fig. 2. 


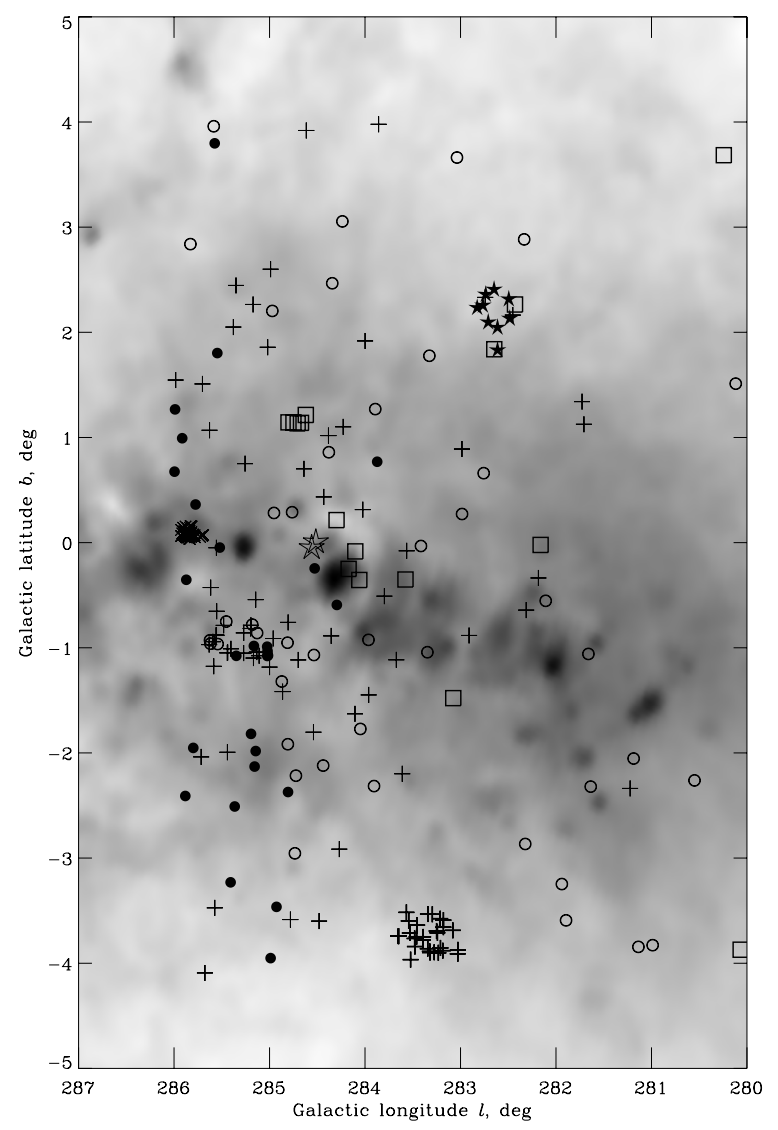

Fig. 5.- All stars with calculated distances and color excesses overplotted on $E(B-V)$ reddening map of the region (Schlegel et al. 1998) obtained via the SkyView VO interface (see McGlynn et al. 1998). The symbols are the same as in Fig. 2. 

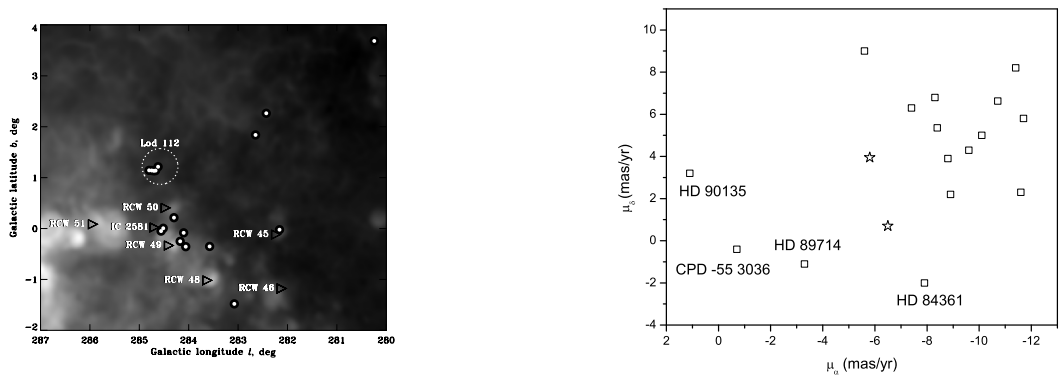

Fig. 6.- (Top). The stars of Lodén 112 and the suggested feature of OB stars overplotted on the distribution of the HiI emission (Finkbeiner 2003) obtained via the SkyView VO interface (McGlynn et al. (1998)). The most prominent HiI regions are labeled. (Bottom). Proper motions from the recalculated Hipparcos catalog for these stars. The two possible members of IC 2581 are marked with asterisks. 

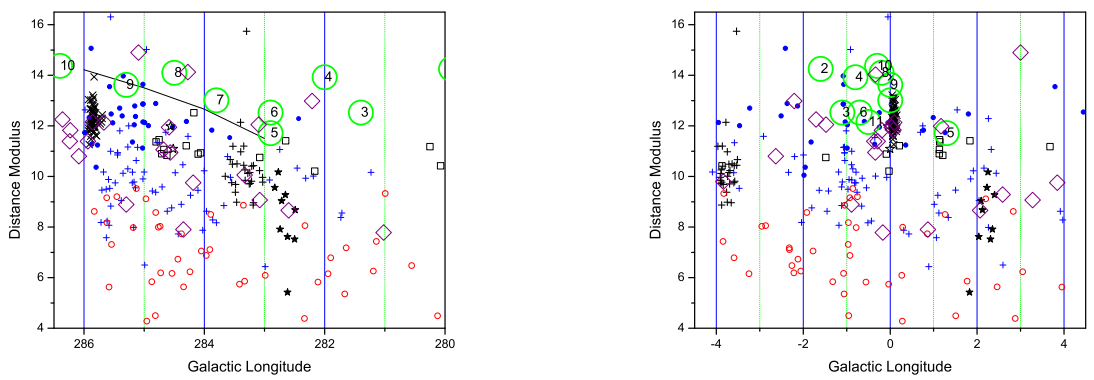

Fig. 7.- (Top). Distance modulus vs. Galactic longitude for the sample stars (the symbols for the stars are the same as Fig. 2). The solid line is the relation obtained by Graham (1970) for the edge of the Carina arm. The large open symbols (labeled) are the GMC from the study of Grabelsky et al. (1988). The clusters and cluster candidates in the field are shown with diamonds. For the clusters studied here the newly obtained distances are used. For the rest of the clusters the accepted distances (Table 2) are utilized. (Bottom). Distance moduli vs. Galactic latitude for the same objects. See the electronic edition of the PASP for a color version of this figure. 